\title{
On the Application of Vagueness in Business Negotiation
}

\author{
Tengyao Zhang \\ Shangqiu University, Henan, China \\ 1292097582@qq.com
}

Keywords: Vagueness; Cooperative principle; Business Negotiation; Pragmatic Functions

\begin{abstract}
Various researches have been done on the ground of vagueness from different perspectives, i.e. philosophically, psychologically, semantically, pragmatically, etc. Vagueness as an important part of linguistic study has been extensively discussed in China. As most scholars agree that vagueness is a useful and necessary Strategy in business negotiation, however few of the studies attempt to explore the vague language in business negotiation systemically from the Pragmatic perspective, this thesis, makes an analysis of vagueness in business negotiation from Grice's cooperative principle. This paper first introduces vagueness with respect to its origin as well as definitions, and then mainly focuses on flouting the maxims of $\mathrm{CP}$ by Vagueness with examples. At last it discusses Pragmatic Functions of Vagueness in Business Negotiation. It also discusses the negative factors in business negotiation and come to the conclusion that this attribute of language should be treated scientifically, not to be ignored and not to be exaggerated.
\end{abstract}

\section{Introduction}

Nowadays international business is playing a more and more important role in our daily life. Business English, both as information and a culture carrier, has been proved an important medium in the international business communication. So the way how people can speak business English not only exactly but also appropriately in order to carry on the business communication effectively and harmoniously becomes a hot study.

With proper use of vague expressions, it can mitigate the potential conflict, create an easy atmosphere and build a close relationship between the participants, negotiators can operate quite happily in the purposive communication .Therefore, as an useful technique, vagueness strategy is needed for negotiators actually.

Vague language plays an important role in business negotiation. The study of vague language in business negotiation may be of practical importance in that it is likely to provide guidelines on how to adopt vague expressions appropriately in negotiation, and consequently, help negotiators become more successful in business negotiation.

\section{Cooperative Principle and Vagueness in Business Negotiation}

Some possible conversational moves would be exuded as conversationally unsuitable. We can make a rough general principle which is "to make your conversational contribution such as required".

Generally in business negotiation, the real idea is not allowed to be expressed for that any information may be directly related to economic interests. When we speak something which is true, relevant, or informative, Cooperative Principle and its maxims usually appear in our mind sub-consciously or even unconsciously. If every participant can follow these principles in any communication, the sensible and efficient communication can be achieved without any inference provided by listeners and indirect expressions by speakers. But this result is difficult to be true because there is few people who observe the principles. 


\subsection{Flouting the maxim of Quantity by Vagueness}

This quantity maxim requires the message be informative but not over-informative. In fact, owing to some subjective and objective elements, a flouting of quantity maxim often appears. For example:

A: in view of our long-standing business relations, I hope you can give us a special discount of $15 \%$.

B: I know that, but business is business.

We all know that in the negotiation of business, price is playing a very important and sensitive role. In this dialogue, A wants to get more discount of price form B. But B does not directly express his agreement or disagreement. B uses the vague expression "business is business", which is a statutory. At the superficial level, what B said does not contain any useful information. However, at a deeper level, his words are very meaningful. He may convey an implicative that "I know our long-standing business relations, but the price we offered you is very practical, so that your counteroffer is unacceptable" B's vague expression is actually a disagreement of refusal. But if B refuses A directly and says: "No, I cannot", A may be easily ruffled and the negotiation may come to a deadlock.

What the example tell us is that the right amount of information can only be valid under a particular context or a particular purpose. That is why many researchers hold that we must add "for the purpose of that conversation".

\subsection{Flouting the maxim of Quality by Vagueness}

Flouts that exploit the maxim of quality occur when the speaker says something which is blatantly untrue in business negotiations. Negotiators obtain particular implications through flouting the maxim, for example:

A: I'm sorry to hear that. But we still find no way to accept your quotation.

B: sorry, it is the last straw of our company, you know.

At the level of what is said, B's answer is a false statement. He flouts the first maxim of quality maxim: "do not say what you believe to be false." In fact, there is not a straw in his hand at that time. Why does the speaker say something false? Of course, the speaker does not want to cheat the hearer. The vague word phrase will not be taken literally. Instead it will be interpreted as a metaphor, which implies "I will not make a concession anymore."

\subsection{Flouting the maxim of Quantity by Vagueness}

The maxim of relevance requests the information to be relevant to the conversation or the topic. If a speaker is giving a response irrelevant to the topic like changing the subject abruptly, we can say that he is flouting the maxim of relevance. For example:

A: Do you think that your delay in delivering goods to us will lead to a great loss?

$\mathrm{B}$ : the weather report says that the typhoon would last a week.

In this dialogue, $\mathrm{A}$ is complaining the great loss caused by B's delay. But B does not give a direct answer to A. It seems that B does not answer the question of $\mathrm{A}$, and he changes to talk something about the weather. But we can easily get the answer form B that it is the weather which leads to the delay of delivery because the typhoon lasts such a long time that the traffic is affected.

\subsection{Flouting the maxim of Manner by Vagueness}

The maxim of manner requests the information given by speakers being clearly, unambiguously and briefly rather than vague and ambiguous. However, negotiators violate it in some cases. For example:

A: The lowest price we can offer is 60 dollars per kilogram. What's your opinion?

B: Not so good, not so bad. We will have a careful consideration about it

In this dialogue, B makes his answer or judgment deliberately uncertain using vague language. In spite of the confusing of A from the answer of B, B has still successfully protected his own business benefits and achieved the business purpose in this negotiation. 


\section{Pragmatic Functions of Vagueness in Business Negotiation}

Vagueness is an inevitable concomitant of ordinary linguistic communication. With proper use of vague expressions, negotiators can operate quite happily in the purposive communication. Vagueness can turn out to be one of negotiation strategies. However, the positive and negative functions of vagueness are context-bounded. A vague expression that brings positive effect in one communication context may bring bad effect in other communicative context. Under some contexts vague expressions are used to confuse the negotiators intentionally, which may give rise to misunderstanding or cheating. So the functions of vague language and the positive and negative role of vague language in business negotiation will be explored in the following.

\subsection{The Positive Role of Pragmatic Vagueness in Business Negotiation}

No matter what kind of vagueness a propositional content may have, it is of positive significance. Sometimes we even find that this "positive murkiness" would be more appropriate in discourse to express a vague idea. The positive function of vagueness in business negotiation is as follows:

\subsubsection{Creating a Harmonious Atmosphere}

Vague language is often used to mitigate the potential conflict, create an easy atmosphere, etc. There are some examples in which various kinds of vagueness are presented.

A: To be frank, \$230 is a fairly high price for your materials, and I am afraid it is hard for me to make it higher.

B: Yes, I can understand that and I hope we have more chances to sit here later in future.

On the one hand, Both A and B in this case are striving for a harmonious atmosphere. What A uses to reduce the influence which is brought by his complain about the high price is "fairly high" and "I am afraid". On the other hand, to indirectly express the refusal of lowering the Price, B leaves enough space for co-operation with each other in the future by saying "have more chance to sit here".

\section{1. 2 Being Flexible and Tactful}

A principal feature of vagueness is indeterminacy. Vague language is relatively elastic and flexible. Vagueness can be employed to minimize categorical commitments. So, the negotiators prefer to loose statements that can be interpreted in a variety of way, thus holding an image of neutrality of sensitive issues and lessening responsibilities.

Vague language can reinforce the flexibility of expressions. Due to the limitations of subject matter, linguistic context and other internal and external factors, we usually avoid definite and blunt expressions by using vague language. Here is an example:

A: This is our rock - bottom price, Mr. Li, we can't make any further concession.

B: If that's the case, there's not much point in further discussion. We might as well call the whole deal off.

Obviously, part A uses exact words "rock bottom price ", the negotiation atmosphere freezes at once. In order to retrieve the nervous situation, A has to explain as follows:

"what I mean is that, we will never be able to cut down to your price . The gap is too great." Even though A resorts to vagueness of language to repair the disadvantage position, no progress will be achieved. So we can see that language without flexibility can lead the negotiation to deadlock. And also in business negotiation, negotiators can make use of vague language to convey meaning in order not to stop the conversation.

If A firstly says "the gap is too great. I hope both of us take some concession." instead of using "rock bottom price", A will have more chance to take this deal. At least it will not cause this deadlock. 3.1.3 Being Self-protecting

Vagueness may be used as a safeguard against being later shown to be wrong. Implications arise, one of which is "speaker is being defensive". It is the same with business negotiation. When negotiators meet the questions which relate to their own benefits, vague words are often employed in the negotiation. Look at this example: 
B: In the tea trade everybody knows that China's black tea is often of top quality .Considering the quality, I should say the price is reasonable.

A: No doubt yours is of a high quality, but still, there is keen competition in the tea market . 1 understands some countries are actually lowering the prices.

B: So far our commodities have stood the competitions well. Few other teas can compare with ours either for favor or color.

A: But I believe we will have a hard time convincing our clients at your Price.

In this example, "of high quality" and "few other teas" are all vague words. A changes "of top quality" into "of high quality", which shows his own opinion and at the same time suggests that even though his tea has high quality but it is not the best one. Meanwhile, it can also avoid the deadlock by saying "you are not the best one in the market." A tries to let B low the price in order to make profits.

\subsection{The Negative Role of Pragmatic Vagueness in Business Negotiation}

It is worth pointing out that one should always keep the point in mind that the importance of vagueness of language cannot be exaggerated. However, too many vague terms in lexical definitions will cause over vague information that people cannot understand, which consequently destroy the basic fiction of a dictionary and make readers feel confused at the real meaning of it.

Business negotiation is an oral communication. Under the same context, the negotiators often employ vague expressions in order to achieve certain purposes. Even though vague languages can work as speech strategies to make negotiation smooth and effective, they can also cause pragmatic failure if they are not used appropriately. Excessive vagueness will leave opposite part the impression of no sincerity to co-operate, which will damage their desire to continue the negotiation. What matters is how to use the vague language appropriately.

As the positive and negative functions of vagueness are context-bounded, therefore this attribute of language should be treated scientifically: not to be ignored and not to be exaggerated.

\section{Conclusion}

In summary, this paper has made tentative attempts to make analyses concerning vagueness in business English negotiation, theoretically based pragmatic principles. It has opened up a new perspective to the study of business English negotiation.

Firstly, the paper introduces vagueness. It is mainly about the origin and the definitions of Vagueness were given by many scholars. And then Cooperative Principle and Vagueness in Business Negotiation was be analyzed by the clue of the Flouting the maxim of CP by Vagueness. Finally, the paper emphasizes Pragmatic Functions of Vagueness in Business Negotiation by making an analysis of it on positive and negative aspects.

Bearing the knowledge in mind does not mean we can deal with the business English negotiation successfully. Negotiation is a complicated process which involved by many factors. To be a good negotiator, firstly he should have some basic economic and legal knowledge which proved to be very useful in the negotiation process. What's more, he is expected to fully consider other factors in culture-specificity, psychology, mode of thinking and opposite party so as to help fulfill the negotiation purpose.

\section{References}

[1] Channell, J. Vague language. Oxford: oxford university press, 2000.

[2] Ullmann, S. Semantics. Oxford: Blackwell, 1962.

[3] Levinson, S. C. Pragmatics. Cambridge: Cambridge University Press, 1987.

[4] Grice, H.P. Syntax and semantics, New York: Academic Press, 1975. 